\title{
Geometry-Based Optimal Power Control of Fading Multiple Access Channels for Maximum Sum-Rate in Cognitive Radio Networks
}

\author{
Wei Wang, Member, IEEE, Wenbo Wang, Member, IEEE, Qianxi Lu, Student Member, IEEE, \\ Kang G. Shin, Fellow, IEEE, and Tao Peng, Member, IEEE
}

\begin{abstract}
In this letter, a power-control scheme for maximum sum-rate is proposed for the fading multiple access channels by considering the presence of primary users. Both the average transmit-power constraints and the peak interferencetemperature constraints are considered. The interference caused by cognitive users must be under a pre-specified threshold for protecting primary users. The power-control optimization is considered as a novel geometrical problem which investigates the relationship of positions of a line and a few points. At most two users transmit simultaneously for optimality and the corresponding conditions are provided for both cases. Based on the analysis, the optimal power control is given for each specific fading state. For lowering computational complexity, the power-control optimization problem is divided into two categories according to different tight constraints. Simulation results are provided for the optimal power-control performance.
\end{abstract}

Index Terms-Cognitive radio, multiple access channel, power control, fading channel, capacity.

\section{INTRODUCTION}

C OGNITIVE radio (CR) [1] is an emerging technology for wireless communications. Unlicensed users equipped with CR are allowed to use the unoccupied licensed spectrum. Several researchers investigated the fundamental limits of CR networks. For example, in [2] and [3], the cognitive user can obtain and transmit the messages that the primary user would like to send. Based on these, [4] analyzes the capacity of a cognitive user who transmits simultaneously with a primary

Manuscript received June 9, 2009; revised December 21, 2009; accepted March 15, 2010. The associate editor coordinating the review of this letter and approving it for publication was S. Affes.

W. Wang is with the Department of Information Science and Electronic Engineering, Key Lab of Integrated Information Network Technology, Zhejiang University, P.R. China. He was with Beijing University of Posts and Telecommunications (BUPT), P.R. China (e-mail: wangw@zju.edu.cn). The work was supported by the National 973 Program under grant No. 2009CB320405, and the National Natural Science Foundation of China under grant No. 60802012 , 60972057 and 60972058, Chinese Universities Scientific Fund under grant No. 2009QNA5029, the Scientific Research Fund of the Zhejiang Provincial Education Department under grant No. Y200909796, and the BUPT PhD Innovation Foundation. His work was partly done during his Ph.D. study in BUPT and his visit to RTCL of the University of Michigan.

W. Wang, Q. Lu, and T. Peng are with the Wireless Signal Processing and Network Lab (WSPN), Beijing University of Posts and Telecommunications (BUPT), P.R. China. Their work was sponsored by the Nokia Corporation and in part by the National Natural Science Foundation of China under grant No.60572120 and 60602058, the National 863 Program under grant No. 2006AA01Z257, and the National 973 Program under grant No. 2007CB310602.

K. G. Shin is with the Real-Time Computing Laboratory (RTCL), Department of Electrical Engineering and Computer Science (EECS), the University of Michigan, Ann Arbor, MI, USA. His work was supported by the U.S. National Science Foundation (NSF) under Grants CNS-0519498 and CNS0721529, and Intel Corporation.

Digital Object Identifier 10.1109/TWC.2010.06.090868 user, if the primary user can achieve the data rate just as it would in the absence of the cognitive user. [5] extends the results of [4] to multiple access channels (MAC) and proposes a heuristic scheme to achieve the maximum sumrate. Unlike the above approaches, [6] analyzes the capacity of a cognitive user under the assumption that the cognitive user cannot obtain the primary users' messages exactly, but only estimate its interference to the primary user via various fading channels. The capacity of a cognitive source-destination pair is investigated considering the average and peak interference constraints respectively. Besides these fundamental researches, some recent works focus on the capacities in more complex scenarios considering MIMO [7] and sensing error [8].

In this letter, we consider the fading multiple access channel in CR networks with the same assumptions in [6]. Different from [6], both the average transmit-power constraints (ATPC) and the peak interference-temperature constraint (PITC) are considered simultaneously. We transform the transmit power to the ratio of the resultant interference to the interferencetemperature limit. Based on this transformation, the optimal conditions derived by the Lagrangian duality are modeled as the relationship between points and lines in a two-dimensional space. From this geometrical model, at most two users are found to be able to transmit simultaneously for optimality. An optimal power-control scheme is proposed for maximum sumrate. The existence of the optimal solution achieved by the proposed scheme is proved from a geometrical respective. The rules are proposed for determining which users are transmitting and how much power they use. Based on these analysis, one of the multiplier can be obtained in a deterministic manner for lower complexity. This problem is divided into two categories according to different tight constraints to decrease the computational complexity further.

The rest of the letter is organized as follows. In Section II, the system model and the capacity region are presented. In Section III, the maximum sum-rate optimization problem is modeled geometrically. In Section IV, a power-control scheme is proposed based on the geometrical model. Following this, the numerical results are provided in Section V. Finally, this letter concludes with Section VI.

\section{Fading Multiple Access Channels in Cognitive RADIO NETWORKS}

Consider a MAC channel scenario in which $N$ cognitive users communicate with the same receiver. The information theory model is given in Fig. 1. The received signal of fading 


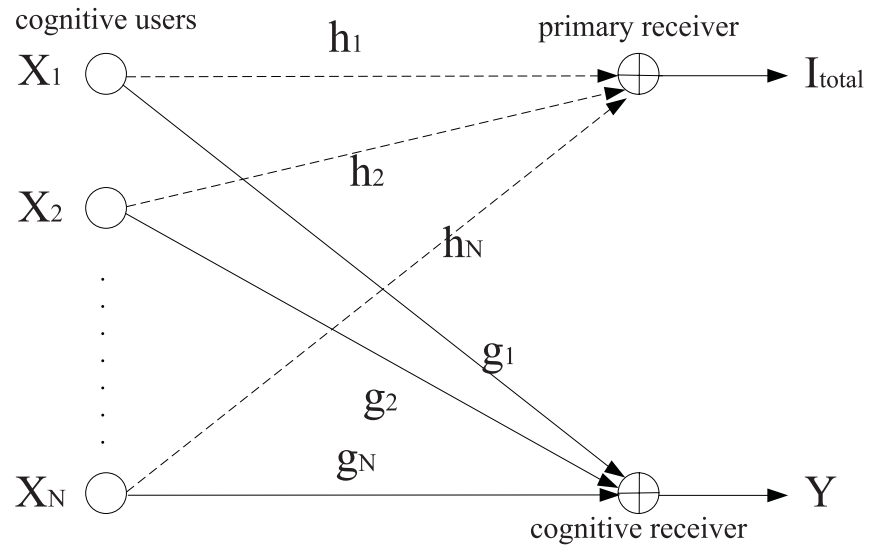

Fig. 1. Information theoretic model of the MAC channel in cognitive radio networks.

MAC channels is characterized as

$$
Y=\sum_{i=1}^{N} \sqrt{g_{i}} X_{i}+Z
$$

where $X_{i}$ is the transmitted waveform of user $i, g_{i}$ is the fading path gain from user $i$ to the receiver, and $Z$ is additive white Gaussian noise (AWGN) noise with variance $\sigma^{2}$.

We consider two types of constraints, ATPC and PITC. Each user's power $P_{i}$ is constrained by an average transmit-power limit $\bar{P}_{i}$.

$$
\mathbb{E}\left[P_{i}\right] \leq \bar{P}_{i} \quad i=1,2, \cdots, N \quad(\text { ATPC })
$$

In CR networks, cognitive users are not allowed to generate too large an interference for protecting primary users. Therefore, interference-temperature constraints are imposed by FCC to limit the interference under a threshold $Q$.

$$
\sum_{i=1}^{N} P_{i} h_{i} \stackrel{\text { a.s. }}{\leq} Q \quad \text { (PITC) }
$$

where $Q$ is the maximum tolerable received power at the primary receiver and $h_{i}$ is the path gain from the user $i$ to the primary receiver. The PITC constraint needs to be satisfied under all possible fading conditions. The channel information can be obtained by receiving the feedback or estimate the received signal strength. For the dummy primary receivers, the interference is restricted at the edge of the service region of primary transmitters.

Define $\mathbf{G}$ as the path gain vector including $g_{1}, \cdots, g_{N}$ and $h_{1}, \cdots, h_{N}, \mathbf{P}(\mathbf{G})$ is the power-control scheme for path gain G. The achievable rate region of the MAC channels is

$$
\begin{gathered}
\mathbb{C}=\bigcup_{\mathbf{P}(\mathbf{G}) \in \mathscr{F}} C(\mathbf{P}(\mathbf{G})) \\
C(\mathbf{P}(\mathbf{G}))=\left\{\begin{array}{r}
\mathbf{R}: R(S) \leq \mathbb{E}\left[\frac{1}{2} \log \left(1+\frac{\sum_{i \in S} P_{i}(\mathbf{G}) g_{i}}{\sigma^{2}}\right)\right], \\
\forall S \subset\{1, \cdots, N\}
\end{array}\right\}
\end{gathered}
$$

where $\mathscr{F}$ is the set of power-control schemes that satisfy the constraints (2) and (3), $S$ is a set of users, $R(S)$ is the sum rate of the users in $S, \mathbf{R}$ is the rate vector of all users.

\section{Geometrical Analysis for MaXimum Sum-Rate PROBLEM}

The goal of this letter is to determine each user's transmit power for all possible fading channel status so as to maximize the sum-rate $R$ under both ATPC (2) and PITC (3) constraints. Let $P_{i}$ substitutes $P_{i}(\mathbf{G})$ for simplicity of expression, the power-control optimization can be formulated as a nonlinear optimization problem.

$$
\begin{array}{ll}
\max & R=\mathbb{E}\left[\frac{1}{2} \log \left(1+\frac{\sum_{i=1}^{N} P_{i} g_{i}}{\sigma^{2}}\right)\right] \\
\text { s.t. } & \mathbb{E}\left[P_{i}\right] \leq \bar{P}_{i} \quad i=1,2, \cdots, N \\
& \sum_{i=1}^{N} P_{i} h_{i} \stackrel{\text { a.s. }}{\leq} Q
\end{array}
$$

\section{A. Lagrangian Optimality Conditions}

The optimal solution of the above problem can be achieved if and only if there exist $\boldsymbol{\lambda}=\left\{\lambda_{1}, \cdots, \lambda_{N}\right\}$ and power-control scheme $\mathbf{P}$ such that the power-control scheme is a solution of the optimization problem for each fading state [9].

$$
\begin{aligned}
\max \quad & \frac{1}{2} \log \left(1+\frac{\sum_{i=1}^{N} P_{i} g_{i}}{\sigma^{2}}\right)-\sum_{i=1}^{N} \lambda_{i} P_{i} \\
\text { s.t. } & \mathbb{E}\left[P_{i}\right] \leq \bar{P}_{i} \quad i=1,2, \cdots, N \\
& \sum_{i=1}^{N} P_{i} h_{i} \leq Q .
\end{aligned}
$$

To achieve the optimal performance, $\boldsymbol{\lambda}$ is adjusted to let the transmit power $\mathbf{P}$ satisfy ATPC constraints. The Lagrangian multiplier $\lambda_{i}$ of user $i$ is unique for all the fading states.

Define $\omega_{i}$ as the ratio of the interference $P_{i} h_{i}$ caused by user $i$ to the maximum tolerable received power $Q$ at the primary receiver, $0 \leq \omega_{i} \leq 1$. In such a case, $\omega_{i} Q / h_{i}$ can be used to represent $P_{i}$ for user $i$. We adjust $\omega_{i}$, instead of $P_{i}$, for power control, then the constraint (11) can be rewritten as

$$
\sum_{i=1}^{N} \omega_{i} \leq 1
$$

Using the Karush-Kuhn-Tucker (KKT) condition, based on Eq. (12), the problem can be transformed to

$$
\begin{aligned}
& \max L= \frac{1}{2} \log \left(1+\frac{\sum_{i=1}^{N} \omega_{i} Q g_{i} / h_{i}}{\sigma^{2}}\right) \\
&-\sum_{i=1}^{N} \lambda_{i} \omega_{i} Q / h_{i}-\beta\left(\sum_{i=1}^{N} \omega_{i}-1\right) \\
& \text { s.t. } \beta\left(\sum_{i=1}^{N} \omega_{i}-1\right)=0
\end{aligned}
$$

where $\beta$ is the Lagrangian multiplier for the constraints (11). Unlike $\lambda, \beta$ is determined for each fading state to ensure that interference to the primary receiver satisfies PITC constraint. 
Let $\sigma^{2}$ be the unit of power, the partial derivative of Lagrangian function $L$ to $\omega_{j}$ is

$$
\frac{\partial L}{\partial \omega_{j}}=\frac{Q g_{j} / h_{j}}{1+\sum_{i=1}^{N} \omega_{i} Q g_{i} / h_{i}}-\lambda_{j} Q / h_{j}-\beta
$$

According to optimization theory, based on different cases of optimal $\omega_{j}^{*}$, the conditions of the optimality are

$$
\begin{cases}\left.\frac{\partial L}{\partial \omega_{j}}\right|_{\omega_{j}=0} \leq 0 & \Rightarrow \omega_{j}^{*}=0 \\ \left.\frac{\partial L}{\partial \omega_{j}}\right|_{\omega_{j}=\omega_{j}^{*}}=0 & \Rightarrow 0<\omega_{j}^{*}<1 \\ \left.\frac{\partial L}{\partial \omega_{j}}\right|_{\omega_{j}=1} \geq 0 & \Rightarrow \omega_{j}^{*}=1\end{cases}
$$

User $j$ is transmitting if $\omega_{j}>0$. When $0<\omega_{j}^{*}<1$, the optimal solution is achieved if $\partial L /\left.\partial \omega_{j}\right|_{\omega_{j}=\omega_{j}^{*}}=0$. When $\omega_{j}^{*}=1$, because the Lagrangian multiplier $\beta$ is configurable for each fading state, we can let $\partial L / \partial \omega_{j}=0$ by adjusting $\beta$. Therefore, the optimal solution of transmitting users is $\partial L /\left.\partial \omega_{j}\right|_{\omega_{j}=\omega_{j}^{*}}=0$ for $\omega_{j}>0$.

\section{B. Geometrical Model}

In this subsection, we propose a novel geometrical model to transform the power-control optimization problem to the position relationship problem of points and lines from a geometrical perspective.

We first rewrite the optimal condition of transmitting users in the power-control problem as an expression of a line in two-dimensional space.

$$
\frac{\partial L}{\partial \omega_{j}}=A x_{j}+B-y_{j}=0
$$

where

$$
\begin{gathered}
x_{j}=Q g_{j} / h_{j} \\
y_{j}=\lambda_{j} Q / h_{j} \\
A=\frac{1}{1+\sum_{i=1}^{N} \omega_{i} Q g_{i} / h_{i}} \\
B=-\beta .
\end{gathered}
$$

Considering this problem from a geometrical view, each user $i$ has a corresponding point $S_{i}\left(x_{i}, y_{i}\right)$ in the twodimensional space. Define $\mathcal{S}$ as the set of all points $S_{i}, \forall i$. For given $\lambda_{i}$, the coordinates $x_{i}$ and $y_{i}$ are determinate. The problem is to determine $A$ and $B$, which are the slope and intercept of the line $Y=A x+B$, respectively. In such a case, the problem is transformed to a new geometrical problem on the position relationship of points $S_{i} \in \mathcal{S}$ and the line $Y=A x+B$. The geometrical problem is to find a line $Y=A x+B$ by adjusting $\boldsymbol{\omega}$ to let some of the points $S_{i} \in \mathcal{S}$ on the line and all the other points above the line.

If only one user $j$ transmits at a time instant, meaning that the line $Y=A x+B$ goes through $S_{j}\left(x_{j}, y_{j}\right)$ and all the other points are on the top of the line, the optimal conditions are

$$
\begin{gathered}
y_{j}=A x_{j}+B \\
y_{i}>A x_{i}+B, \forall i \neq j
\end{gathered}
$$

Because $\beta$ can be configured as any desired value, the line $Y=A x+B$ can always go through a point by adjusting the intercept $B$.
If two users $j$ and $k$ transmit simultaneously at a time instant, meaning that the line $Y=A x+B$ go through both $S_{j}\left(x_{j}, y_{j}\right)$ and $S_{k}\left(x_{k}, y_{k}\right)$, and all the other points are above the line, the following equations and inequations should be satisfied.

$$
\begin{gathered}
y_{j}=A x_{j}+B \\
y_{k}=A x_{k}+B \\
y_{i}>A x_{i}+B, \forall i \neq j, k
\end{gathered}
$$

For the case when more than 2 users transmit simultaneously, the following theorem can be obtained from a geometrical view.

Theorem 1: For optimality, at most 2 users can transmit simultaneously.

Proof: Reasoning by contradiction, suppose there exist more than 2 users transmit at the same time. Assume there are 3 users $j_{1}, j_{2}$ and $j_{3}$ without loss of generality, $\omega_{j_{n}}>$ $0, n=1,2,3$. Based on (12), $0<\omega_{j_{n}}<1, n=1,2,3$. For optimality, the following equations need to be satisfied according to the optimality conditions of transmitting users.

$$
\partial L / \partial \omega_{j_{n}}=A x_{j_{n}}+B-y_{j_{n}}=0, \quad n=1,2,3 .
$$

Based on (18) and (19), the coordinates $\left(x_{j_{n}}, y_{j_{n}}\right)$ of the point $S_{j_{n}}$ are interrelated to the path gains $g_{j_{n}}$ and $h_{j_{n}}$, which are continuous variables. A line $y=A x+B$ in a two-dimensional space can be found with probability 0 to go through all the 3 points, which is a contradiction. Therefore, at most 2 users can transmit at the same time.

\section{Optimal Power CONTROL}

\section{A. Geometrical Method for Optimal Solution}

To find a line $y=A x+B$ such that some of the points $S_{i}, \forall i$ on the line and all the other points above the line, we can find a line tangent to the polygon whose vertices are a subset of $\mathcal{S}$ and all the points of $\mathcal{S}$ are inside the polygon. To guarantee all the points either on or above the line, the tangent point should be at the lower side of the polygon. From the point with minimum $x_{i}$, according to the counter-clockwise order, the vertices of the polygon compose a sequence $S_{V_{1}}, S_{V_{2}}, \cdots, S_{V_{E}}$, where $E$ is the number of vertices at the lower side of the polygon, as described in Fig. 2, in which $E=4$.

For investigating the tangency of a line and a polygon, the slopes of the edges of the polygon are analyzed and compared with the slope of the line. Define $L$ denote a piece-wise curve composed with the slopes of the edges at the lower side of the polygon, as only the case that all points in $\mathcal{S}$ are either on or above the line is considered.

$$
L(x)=\arg \tan \frac{y_{V_{i}}-y_{V_{i-1}}}{x_{V_{i}}-x_{V_{i-1}}}, \quad x \in\left[x_{V_{i-1}}, x_{V_{i}}\right) .
$$

Define $M$ as a folding line composed by the slopes of line $y=A x+B$ if it goes through $S_{V_{j}}$ and let all the other points in $\mathcal{S}$ above the line. In such a case, $\omega_{V_{j}}=1$, and for other points $S_{i} \neq S_{V_{j}}, \omega_{i}=0$. Based on the definitions (18) and (20), we get

$$
M\left(x_{V_{i}}\right)=\arg \tan \frac{1}{1+Q g_{V_{i}} / h_{V_{i}}}=\arg \tan \frac{1}{1+x_{V_{i}}} .
$$



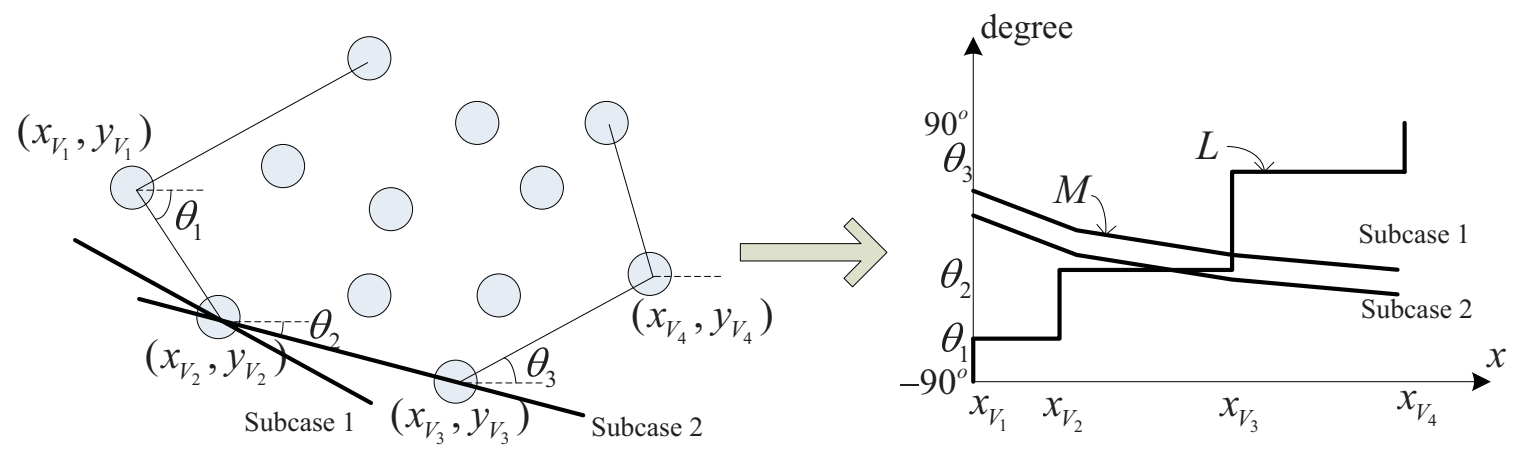

Fig. 2. Geometrical model.

According to the characteristics of the geometrical model, the existence and uniqueness of the optimal solution are obtained as the following theorem.

Theorem 2: A unique solution exists for the optimal power control optimization problem.

Proof: For given $\boldsymbol{\lambda}$, because $x_{i}>0,1 /\left(1+x_{i}\right)<1$, the curve $M$ decreases with the increase of $x_{i}$ within $\left(0,45^{\circ}\right)$. By contrast, $L$ increases from $-90^{\circ}$ to $90^{\circ}$ monotonously. Therefore, a unique intersection of $M$ and $L$ exists, so does the unique solution.

Consider the intersection point of $M$ and $L$ in Fig. 2. By comparing $M(x)$ and $L(x)$ at all the points $x_{V_{1}}, x_{V_{2}}, \cdots, x_{V_{E}}$, the intersection point can be obtained. If the intersection of $M$ and $L$ is at the vertical part of $L$ with $x_{V_{i}}$ (Subcase 1 in Fig. 2), the slope of line $y=A x+B$ at $x_{V_{i}}$ is between the slopes of line $S_{V_{i-1}} S_{V_{i}}$ and line $S_{V_{i}} S_{V_{i+1}}$,

$$
\frac{y_{V_{i}}-y_{V_{i-1}}}{x_{V_{i}}-x_{V_{i-1}}}<\frac{1}{1+x_{V_{i}}}<\frac{y_{V_{i+1}}-y_{V_{i}}}{x_{V_{i+1}}-x_{V_{i}}}
$$

so it is optimal that the user corresponding to $S_{V_{i}}$ transmits. In the other case, if it is at the horizontal part of $L$ between $x_{V_{i}}$ and $x_{V_{i+1}}$ (Subcase 2 in Fig. 2), the slope of line $y=A x+B$ at $x_{V_{i}}$ is larger than the slope of line $S_{V_{i}} S_{V_{i+1}}$ but the slope at $x_{V_{i+1}}$ is less than it,

$$
\frac{1}{1+x_{V_{i+1}}}<\frac{y_{V_{i+1}}-y_{V_{i}}}{x_{V_{i+1}}-x_{V_{i}}}<\frac{1}{1+x_{V_{i}}}
$$

so it is possible to find a line $y=A x+B$ which goes through both $S_{V_{i}}$ and $S_{V_{i+1}}$, and two users corresponding to $S_{V_{i}}$ and $S_{V_{i+1}}$ should transmit at the same time.

Because there are two constraints ATPC and PITC in the problem, two Lagrangian multipliers are needed to transform this problem to another optimization problem without constraint. By the above method, the multiplier $\omega$ can be obtained in a deterministic manner by at most $N$ times of comparison, which decreases the computational complexity a lot compared with the classic multiplier adjustment method (i.e. steepest descent method).

\section{B. Algorithm Description}

Two possible cases are considered for this problem according to whether the PITC constraint is tight or not. A 'tight' constraint means that "=" is satisfied in the inequality constraint.
When the PITC constraint is tight (Case 1), the interference to the primary user is just $Q$. When only one user $j$ transmits, which is determined by (30) in Subcase 1, the transmit power should be $Q / h_{j}$. When two users $j$ and $k$ transmit simultaneously, which is determined by (31) in Subcase 2, the optimal power-control scheme should let line $y=A x+B$ go through both $S_{j}$ and $S_{k}$ as the geometric model. In such a case, the transmit power of user $j$ and user $k$ are $\omega_{j} Q / h_{j}$ and $\omega_{k} Q / h_{k}$ respectively. The parameters $\omega_{j}$ and $\omega_{k}$ can be determined by solving the following equations:

$$
\left\{\begin{array}{l}
\left(y_{j}-y_{k}\right) /\left(x_{j}-x_{k}\right)=1 /\left(1+\omega_{j} x_{j}+\omega_{k} x_{k}\right) \\
\omega_{j}+\omega_{k}=1
\end{array}\right.
$$

When the PITC constraint is not tight (Case 2), ATPC constraints of all users are tight, $\beta=0$ and $\lambda_{i} \neq 0$ $\forall i \in \mathscr{N}$, which can be argued by contradiction. Suppose the PITC constraint is not tight at some time instant, and the ATPC constraint of user $j$ is also not tight for the optimal solution. At this time instant, the transmit power of user $j$ can be increased to achieve larger date rate than the optimal solution, until either the two constraints is tight, which is a contradiction. Therefore, when the PITC constraint is not tight, ATPC constraints of all users must be tight for optimality.

In Case 2, the optimality condition can be simplified as

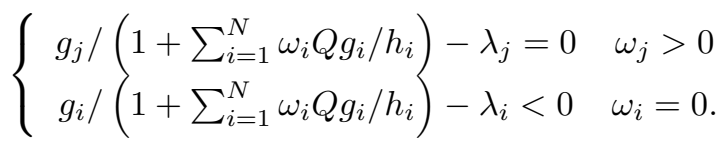

Because the path gain $g_{i}$ is a continuous variable for all $i$, the $g_{i} / \lambda_{i}$ of two users are not equal with probability 1 . Therefore, at most one user transmit in Case 2. Rewrite the optimality conditions as

$$
\left\{\begin{array}{cc}
g_{j} / \lambda_{j}=1+\omega_{j} Q g_{j} / h_{j} & \omega_{j}>0 \\
g_{i} / \lambda_{i}<1+\omega_{j} Q g_{j} / h_{j} & \omega_{i}=0, \forall i \neq j
\end{array}\right.
$$

To satisfy the conditions, the optimal strategy is choosing the user with the largest $g_{i} / \lambda_{i}$ which is user $j$ for instance. The optimal transmit power restricted by ATPC can be obtained by solving

$$
g_{j} / \lambda_{j}=1+P_{j} g_{j} .
$$

Considering that the PITC constraint is tight sometimes, the optimal transmit power is $\min \left\{\frac{Q}{h_{j^{*}}}, \frac{1}{\lambda_{j^{*}}}-\frac{1}{g_{j^{*}}}\right\}$ [12].

If $g_{j}<\lambda_{j}$ for the user $j$ with the largest $g_{j} / \lambda_{j}$, which means that $g_{i}<\lambda_{i} \forall i \in \mathscr{N}$, then $g_{i} / \lambda_{i}<1+\omega_{i} Q g_{i} / h_{i}$ is 
satisfied even if $\omega_{i}=0$. In this case, the channel quality is too bad to transmit for all users.

The power-control problem in both cases can be solved uniformly by comparing $M$ and $L$ at every vertices of the polygon based on the geometric model. However, the problem in Case 2 is much easier than the general case. Therefore, by classification, the computational complexity of the proposed scheme can be decreased. Using this method, only one Lagrangian multiplier $\boldsymbol{\lambda}$ needs to be adjusted, so the algorithm converges while the adjustment steps satisfy the rules provided in [13]. The pseudo codes of the proposed optimal power control is provided as Algorithm 1.

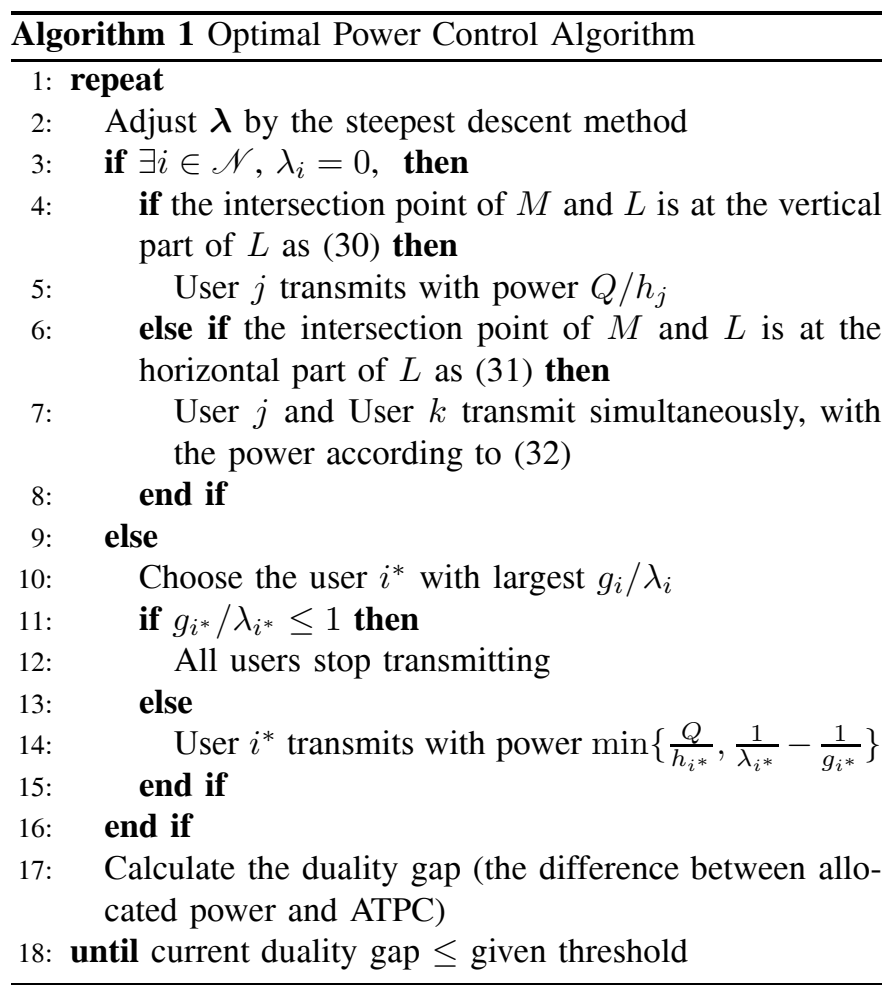

\section{NuMERICAL RESUlts}

To provide an insight into the proposed power-control scheme and the achieved capacity of fading MAC channels in CR networks, a simulator is built for CR networks. In this simulation, 1 primary receiver and 3 cognitive users are distributed randomly in a $30 \mathrm{~km} \times 30 \mathrm{~km}$ square area, and a 1 cognitive base station is located at the center of this area. The path gain is calculated using Free Space Propagation Model. Fading channel model A for IEEE 802.22 WRAN wireless link [14] is applied. The bandwidth of each subcarrier is $3384 \mathrm{~Hz}$ as defined in the WRAN system model [15]. Each cognitive user can transmit with $48 \mathrm{dBm}$ at most. To achieve average performance, the semi-static simulation is executed for 500 topologies with random user locations. For each topology, 100 time slots are considered.

The following numerical results indicate the optimal performance achieved by the proposed power-control scheme. Fig. 3 shows the throughput with different PITC constraints by the ratio to the throughput without any PITC constraint. With large PITC constraints, the throughput in CR networks approaches

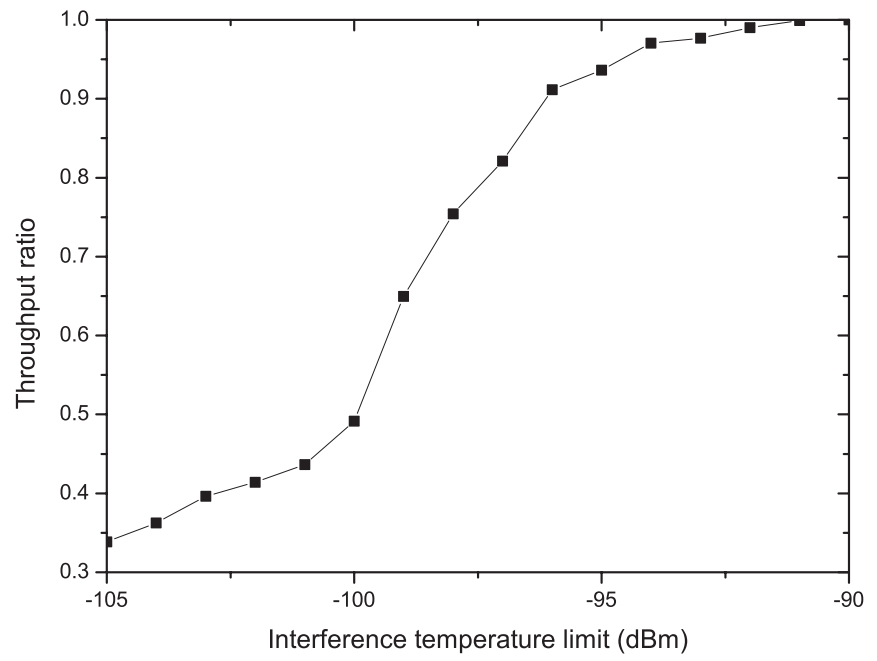

Fig. 3. Ratio of the throughput with PITC constraint to the throughput without PITC constraint.

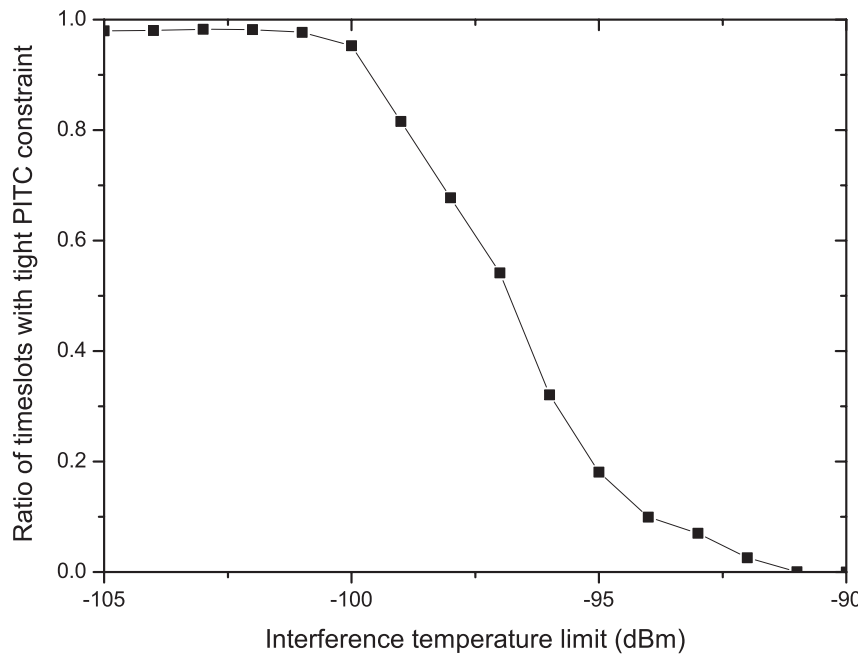

Fig. 4. Ratio of timeslots with tight PITC constraint.

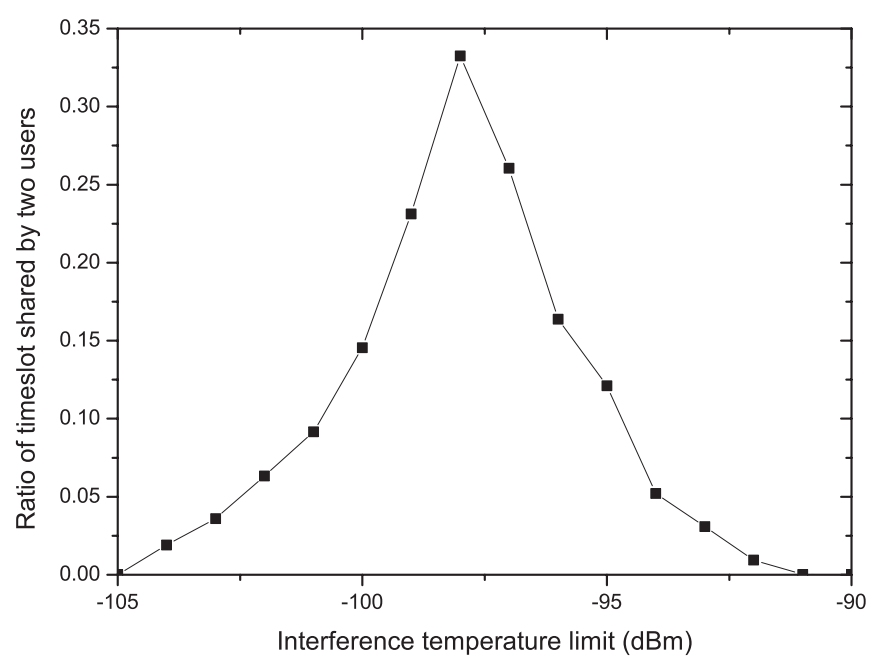

Fig. 5. Ratio of timeslots shared by two users. 
the performance without considering the PITC constraint. This is because the PITC constraint is not tight for most of the time when the PITC limit is large, as shown in Fig. 4. Fig. 5 gives the percentage of the time slots which are shared by two users. We made an interesting observation: two users transmit simultaneously when the PITC constraint is moderately tight.

\section{CONCLUSION}

In this letter, a geometry-based optimal power-control scheme is proposed for fading MAC channels in CR networks. The ATPC constraints and the PITC constraint are considered simultaneously. For this scheme, the properties of the power-control optimization problem are exploited by Lagrangian duality. Based on these properties, this problem is transformed to a novel geometrical problem which explores the position relationship between lines and points. At most 2 users are allowed to transmit at the same time for optimality and the corresponding conditions are provided. The optimal power-control scheme is proposed based on the geometrical model. To decrease the computational complexity, one of the Lagrangian multiplier is obtained by the deterministic method and the problem is divided into two categories according to different tight constraints. By the proposed scheme, the optimal power-control solution is demonstrated via numerical evaluation.

\section{REFERENCES}

[1] J. Mitola and G. Maguire, "Cognitive radio: making software radios more personal," IEEE Personal Commun. Mag., vol. 6, no. 4, pp. 13-18, Aug. 1999.
[2] N. Devroye, P. Mitran, and V. Tarokh, "Cognitive multiple access networks," in Proc. IEEE ISIT 2005, pp. 57-61, Sep. 2005.

[3] N. Devroye, P. Mitran, and V. Tarokh, "Achievable rates in cognitive radio channels," IEEE Trans. Inf. Theory, vol. 52, no. 5, pp. 1813-1827, May 2006.

[4] A. Jovicic and P. Viswanath, "Cognitive radio: an information-theoretic perspective," in Proc. IEEE ITST 2006, pp. 2413-2417, May 2006.

[5] P. Cheng, G. Yu, Z. Zhang, H. Chen, and P. Qiu, "On the achievable rate region of Gaussian cognitive multiple access channel," IEEE Commun. Lett., vol. 11, no. 5, pp. 384-386, May 2007.

[6] A. Ghasemi and E. Sousa, "Fundamental limits of spectrum-sharing in fading environments," IEEE Trans. Wireless Commun., vol. 6, no. 2, pp. 649-658, Feb. 2007.

[7] S. Sridharan and S. Vishwanath, "On the capacity of a class of MIMO cognitive radios," IEEE J. Sel. Topics Signal Process., vol. 2, no. 1, pp. 103-117, Feb. 2008.

[8] Y. Chen, Q. Zhao, and A. Swami, "Joint design and separation principle for opportunistic spectrum access in the presence of sensing errors," IEEE Trans. Inf. Theory, vol. 54, no. 5, pp. 2053-2071, May 2008.

[9] D. Tse and S. Hanly, "Multiaccess fading channels - part I: polymatroid structure, optimal resource allocation and throughput capacities," IEEE Trans. Inf. Theory, vol. 44, no. 7, pp. 2796-2815, Nov. 1998.

[10] R. Knopp and P. Humblet, "Information capacity and power control in single-cell multiuser communications," in Proc. IEEE ICC 1995, pp. 331-335, June 1995.

[11] M. Khojastepour and B. Aazhang, "The capacity of average and peak power constrained fading channels with channel side information," in Proc. IEEE WCNC 2004, pp. 77-82, Mar. 2004.

[12] W. Wang, W. Wang, Q. Lu, and T. Peng, "An uplink resource allcoation for OFDMA-based cognitive radio networks," Wiley Int. J. Commun. Sys., vol. 22, no. 5, pp. 603-623, May. 2009.

[13] K. Kar, S. Sarkar, and L. Tassiulas, "A simple rate control algorithm for maximizing total user utility," in Proc. IEEE Infocom 2001, Apr. 2001.

[14] S. Kuffner, "Channel models with non-integer delays," IEEE 802.22-07/0175r0, 2007. [Online]. Available: http://www.ieee802.org/22/Meeting-documents/2007-May.

[15] C. Cordeiro, "A cognitive MAC (CMAC) proposal for IEEE 802.22 WRAN systems," IEEE 802.22-05/0105r0, 2005. [Online]. Available: http://www.ieee802.org/22/Meeting-documents/2005-Nov. 